\title{
Nanostructured porous polymer monolithic columns for capillary liquid chromatography of peptides
}

\author{
Axel Vaast ${ }^{1}$, Herman Terryn ${ }^{2}$,Frantisek Svec $^{3}$, Sebastiaan Eeltink ${ }^{1, *}$ \\ ${ }^{1}$ Vrije Universiteit Brussel, Department of Chemical Engineering, Brussels, Belgium \\ ${ }^{2}$ Vrije Universiteit Brussel, Department of Materials and Chemistry, Brussels, Belgium \\ ${ }^{3}$ The Molecular Foundry, E.O. Lawrence Berkeley National Laboratory, Berkeley, CA, USA
}

(*) corresponding author

Pleinlaan 2, B-1050, Brussels, Belgium

Tel.: +32 (0)2 629 3330, Fax: +32 (0)2 629 3248, E-mail: seeltink@ vub.ac.be 


\section{ABSTRACT}

The macroporous structure of poly(styrene-co-divinylbenzene) monolithic capillary columns has been optimized for the gradient separation of peptides. To exploit monolithic supports with porosity exceeding $70 \%$, the thermodynamic properties of the polymerization mixture were carefully tailored to yield homogeneous monolithic materials featuring macropore and polymer microglobule sizes in the range of 50-200 nm. The effects of (i) initiator content, (ii) composition of porogenic mixture, comprising tetrahydrofuran and 1-decanol, (iii) percentage of divinylbenzene crosslinker, and (iv) monomers to porogen ratio on the morphology was investigated. The resulting column structures were investigated using scanning electron microscopy and the prepared monolithic columns were tested for the separation of a tryptic digest of cytochrome $c$ while applying a fixed flow rate and gradient time. To obtain a better understanding of the effects of macropore and microglobule size, and structure homogeneity on the separation performance in gradient elution, both in terms of peak capacity and gradient plate height, separations were also carried out at different flow rates while maintaining a constant gradient steepness. Furthermore, performance limits were determined applying ultra-high pressure conditions up to the maximum system pressure of $80 \mathrm{MPa}$. The potential of monolithic nanostructured columns is demonstrated for the separation of tryptic digests of cytochrome $c$ and bovine serum albumin

Keywords: Poly(styrene-co-divinylbenzene); Morphology; Ballistic gradients; capillary UHPLC; Kinetic plot; Gradient van Deemter 


\section{Introduction}

One of the first discussions related to the prospects of polymer-monolithic stationary phases for liquid chromatography (LC) can be traced back to the late 1940s with Tiselius, Martin, and Synge [1]. They envisioned a single interconnected polymeric network which would allow a solvent to percolate through the distinct macropores. However, early forms of such polymeric materials collapsed due to their gel-like appearance when hydrostatic pressure was applied to generate a flow, thus making them unsuitable for chromatographic applications. The surge in interest in monolithic materials as versatile, interconnected support structures for flow-through applications was only regained after the development of rigid polymer-based monolithic materials in the 1990s [2,3]. From then on, monolithic structures could be implemented as stationary phase supports for high-performance liquid chromatographic separations, as carriers for (bio)catalysis, and in many other applications.

The two most prominent classes of rigid polymer-monolithic materials are the methacrylate-ester-based monoliths and monolithic stationary phase prepared from styrene and divinylbenzene precursors [4,5]. A considerable advantage of such poly(styrene-codivinylbenzene) monolithic materials is their chemical stability, both at low and high $\mathrm{pH}$. Their chromatographic potential in large internal diameter columns has been demonstrated for the separation of large molecules, such as synthetic polymers [6] and intact proteins [7]. Life-science applications, which often require the hyphenation with electrospray ionization-mass spectrometry (ESI-MS), further spurred the development of monoliths in narrow-bore column format. In this context, capillary columns were successfully used for the profiling of complex proteomic samples, such as for the baseline separation of single- and double-stranded nucleic acid [8], and the mass fingerprinting of tryptic peptides [9]. Recently, our group also emphasized the 
versatility of monolithic capillary column technology for both high-resolution separations of peptides [10] and intact proteins (including protein isoforms resulting from various amino-acid modifications) [11], and high-speed gradient UHPLC separations of tryptic digests and intact proteins [12].

The preparation of porous polymer entities is typically based on the single-step freeradical polymerization of mono- and oligovinylic monomers in the presence of a porogen using thermal initiation $[13,14]$. The relative composition and nature of the precursor mixture prior to polymerization largely affects the final morphology of the monolithic structure. Most monolithic materials have been prepared following the original recipe described by Svec and Fréchet, i.e. using $40 \mathrm{wt} \%$ monomers and $60 \mathrm{wt} \%$ porogens (as well as $1 \mathrm{wt} \%$ initiator with respect to the monomer content) [15]. The porogens are selected based on their ability to dissolve the monomers, to enable the polymer phase separation process, and to support the formation of a macroscopic porous network during polymerization. When the molecular weight of the polymer formed increases, a phase separation is induced and the reticulated three-dimensional macroporous polymer network emerges [16]. By adjusting the porogen ratio, e.g., by using a binary porogen system (principle of "good" and "bad" solvent) [17], the macroporous properties can be optimized. These properties affect the magnitude of the eddy dispersion $(A$-term) and the resistance to mobile-phase mass-transfer $\left(C_{m}\right.$-term). Due to a limited presence of mesopores (defined as stagnant pores with a size of 2-50 nm), organic polymer-based monolithic materials typically exhibit a small surface area in the range of $10-50 \mathrm{~m}^{2} / \mathrm{g}$, as measured in the dry state [13]. The lack of mesopores results, for large-molecule separations, that the diffusion-limited mass-transfer contribution $\left(C_{s}\right.$-term) to band broadening, as encountered for small-molecule separations, becomes virtually absent $[18,19]$. 
Kinetic plots, visualizing the effect of morphology on efficiency and analysis time have spurred the development of column technology [20]. Generally, smaller characteristic sizes (i.e., domain size) of the chromatographic bed are renowned to reduce the plate-height values, and hence increase the column efficiency. However, this occurs at the cost of a decrease in column permeability. It has been demonstrated that for self-similar bed structures, such as columns packed with particles differing in size [21], different regions in the kinetic plot can be distinguished [20, 22-24]. Short columns packed with small particles provide the best trade-off between analysis time and efficiency for high-speed separations, whereas long columns packed with larger particles enable superior (high-resolution) separations, though in combination with long analysis times. While the efficiency of packed columns is related to the particle size, the drawback is that the external porosity is fixed. Monolithic stationary phases could in that aspect have the potential to perform intrinsically better than packed columns, since porosity can be finetuned based on the total content of monomers and porogens in the initial polymerization mixture. By developing highly porous monolithic structures, longer columns could be used, which in turn could yield higher separation efficiency. However, to exploit high-porosity monolithic supports at a practically relevant range of analysis times, the macropore and globule size needs to be carefully tuned (i.e., decreased), while the structural homogeneity must be maintained.

The present study concerns the preparation and characterization of high-porosity poly(styrene-co-divinylbenzene) monoliths in capillary column format, featuring different (nano)pore structures. The thermodynamic properties of the polymerization mixture have been systematically optimized to tune both the size of the macropores and the microglobules forming the polymer matrix. The properties of the resulting monolithic materials have been assessed with scanning electron microscopy and the chromatographic performance for the separation of 
peptides has been tested for selected columns in gradient-elution mode. Finally, the potential of monolithic nanostructured materials in chromatographic separations is demonstrated for the separation of tryptic digests of cytochrome $c$ and BSA

\section{Materials and methods}

\subsection{Chemical and reagents}

Styrene (S, 99.5\%), divinylbenzene (DVB), composed of $m$-DVB and $p$-DVB (together 80\%), 3-(trimethoxysilyl)propyl methacrylate (98\%), 2,2'-azobis(2-methylpropionitrile) (AIBN, 98\%), tetrahydrofuran (THF, anhydrous, without stabilizer, 99.9\%), 1-decanol (99\%), toluene (anhydrous, 99.8\%), aluminium oxide, and uracil (HPCE, 99\%) were purchased from SigmaAldrich (Bornem, Belgium). Hydrochloric acid (30\% v/v) was purchased from Fluka (Buchs, Germany). Acetonitrile (ACN, HPLC supra-gradient quality), acetone (ACS grade, $\geq 99.5 \%$ ), and formic acid (FA, $\geq 99.0 \%$ ) were purchased from Biosolve (Valkenswaard, The Netherlands). Deionized water was produced in-house using a Milli-Q gradient water-purification system (Millipore, Molsheim, France). A 5-peptide standard mixture (Proteomix, PepMix 4) containing bradykinin fragment 1-5, angiotensin II, neurotensin, ACTH clip [18-39], and oxidized bovine insulin $\beta$-chain was purchased from LaserBio Labs (Sophia-Antipolis, France). Tryptic digests of bovine cytochrome $c(1.6 \mathrm{nmol}$, lyophilized) were purchased from Thermo Fisher Scientific (Amsterdam, The Netherlands). Tryptic digests of bovine serum albumin (BSA, 500 pmol, lyophilized) were purchased from Bruker Daltonic (Bremen, Germany)

\subsection{In-situ preparation of monolithic structures}


Fused-silica tubing $(200 \mu \mathrm{m}$ i.d. $\times 360 \mu$ m o.d.), acquired from Polymicro Technologies (Phoenix, AZ , USA), was pretreated to establish a covalent bond between the wall and monolithic structure. Briefly, the capillary wall was initially etched by flushing with $1 \mathrm{~mol} / \mathrm{L}$ $\mathrm{NaOH}$ solution for $30 \mathrm{~min}$. Next, a silanol-regeneration was achieved by flushing with $1 \mathrm{~mol} / \mathrm{L}$ $\mathrm{HCl}$ for $30 \mathrm{~min}$, followed by a functionalization step by pumping 10 vol\% 3 (trimethoxysilyl)propyl methacrylate solution in toluene for $60 \mathrm{~min}$. All these steps were carried out at a flow rate $2 \mu \mathrm{L} /$ min using a syringe pump. Finally, the capillaries were washed with acetone and dried.

Prior to polymerization, S and DVB were purified by passing them over activated basic alumina in order to remove tert-butylcatechol, which serves as storage stabilizer (polymerization inhibitor). As a starting point, the reference polymerization mixture was composed of $30 \mathrm{wt} \%$ monomers (S and DVB cross-linker), $70 \mathrm{wt} \%$ porogen (THF and decanol), and initiator (AIBN, 1 wt\% with respect to the monomers). After mixing and degassing in an ultrasonic bath (both for $10 \mathrm{~min}$ on ice), the polymerization mixture was introduced in the pretreated capillary via a syringe. After sealing both ends of the capillary with a septum, polymerization was carried out in a water bath (Julabo, Seelbach, Germany) at $70^{\circ} \mathrm{C}$ for $24 \mathrm{~h}$. The monolith was then flushed at a pressure of $20 \mathrm{MPa}$ for $4 \mathrm{~h}$ with $\mathrm{ACN}$ to remove any unreacted species. Compositions of the specific polymerization mixtures are provided in the figure captions.

\subsection{Instrumentation}

Scanning electron microscopy (SEM) was performed in secondary electron imaging mode using a JSM-6400 field emission scanning electron microscopy (JEOL, Tokyo, Japan), operated at acceleration voltages of $10 \mathrm{keV}$. Cross-sections of the columns were sputtered with a $12 \mathrm{~nm}$ 
layer of Pt-Pd using a $208 \mathrm{HR}$ sputter coater equipped with a Cressington mtm 20 Thickness Controller (Cressington Scientific Instruments, Watford, UK).

The chromatographic measurements were carried out using a UltiMate 3000 RSLCnano system (Thermo Fisher Scientific, Germering, Germany), comprising a membrane degasser, a binary high-pressure gradient pump, a capillary flow selector, a thermostated well-plate sampler, a column oven set to $60^{\circ} \mathrm{C}$, and a UV detector equipped with a $3 \mathrm{~nL}$ nano Z-view flow cell. UV detection was performed at $210 \mathrm{~nm}$ with a $50 \mathrm{~Hz}$ data collection rate and $0.03 \mathrm{~s}$ response time. Chromeleon software (version 6.80, SR13) was used for system operation and data evaluation. An on-column injection setup $(1 \mu \mathrm{L})$ was configured to minimize both the gradient-delay volume and external band-broadening effects. Linear ACN gradients were applied from 1 to 40 vol\% B, using a mobile phase A composed of 0.05 vol\% aqueous FA and a mobile phase B composed of 80 vol\% (aqueous) ACN, containing 0.04 vol\% FA. Samples were prepared in a solvent which final composition always matched the initial solvent composition of the gradient (i.e. 1 vol\% B). The solutions of lyophilized 5 peptide mixture, tryptic digest of cytochrome $c$, and BSA digest were prepared in a concentration range of $1-2 \mu \mathrm{g} / \mu \mathrm{L}$.

\section{Results and discussion}

\subsection{Optimization of the macroporous monolithic structure}

High-porosity monolithic polymers were prepared in-situ within the confines of capillaries via the thermally-initiated free-radical crosslinking polymerization using a fixed monomer to porogen ratio of 30:70 wt $\%$. The polymerization reaction was carried out for $24 \mathrm{~h}$ to reach complete conversion [25]. To tailor the size of the macropores and the polymer microglobules in the submicrometer range, the effect of experimental variables on the formation 
of macroporous copolymer networks were investigated by altering the amount of initiator, porogen composition, and crosslinker content. In the first stage of the polymerization reaction, 2cyanoprop-2-yl radicals originating from the thermal decomposition of AIBN are formed homogenously throughout the polymerization mixture. These radicals initiated chain growth and branching. In the next stage, cyclization reactions yielded the formation of compact polymer microspheres (microgelation), which subsequently underwent multiple crosslinking reactions via pendant vinyl groups to form polymer microglobules (macrogelation). Once reaching the threshold molecular weight, phase separation of the polymer occurs. The microglobules swollen with polymerizing monomers continue to grow, and after they coalesce they form the final macroporous three-dimensional structure. The scanning electron micrographs depicted in Fig. 1 illustrate the typical interconnected globular structure of polymer monoliths formed via nucleation and subsequent growth, characteristic for a bimodal phase separation [26]. Lubbad and Buchmeiser demonstrated that initiator content strongly affect the bed structure and hence the separation efficiency [27]. Monoliths were prepared with 1, 3, 5, 7, and 9 wt $\%$ of initiator with respect to the total monomer content. An increased amount of initiator in the polymerization mixture resulted in a significant decrease in globule size, as shown in Fig. 1A-C. This could be attributed to an increase in number of initiating free radicals. As a result, the individual (smaller) globules became more accessible for the mobile phase while displaying a decreased macropore size. However, at even smaller macropore size, domains of larger agglomerates composed of very small globules were formed, affecting the structural homogeneity. This could be observed when increasing the initiator content to $5 \mathrm{wt} \%$ and above, as the homogeneity of the macroporous structure was significantly impaired (Fig. 1C). In this case, porous regions composed of very small interconnected polymer globules accessible to the mobile phase were alternated with large 
domains of polymer agglomerates. In addition, the porous structure was interspersed by large flow-through pores.

Figures 1D-F show micrographs of monoliths prepared from polymerization mixtures with fixed (15 wt $\%$ of both $\mathrm{S}$ and DVB) monomer to porogen ratios of 30:70 wt $\%$ and initiator content (3 wt\%), while varying the porogen composition. THF and 1-decanol were selected as binary porogen system since they constitute solvents for the monomers enabling the preparation of a homogenous polymerization mixture. When the porogen displays good compatibility with the polymer network, the network remains solvated up to a high conversion of monomers in the polymer [22]. Since THF ("good solvent") is more proficient in solvating the polymer network than 1-decanol ("poor solvent"), the phase separation is delayed when the percentage of THF in the porogen is increased. Furthermore, as both the good solvent and monomers solvate the polymer chains, the local monomer concentration in the swollen nuclei is lower than outside and the globules grow to a smaller size. After the phase separation, a polymer network consisting of small interconnected globules is formed, as presented in Fig. 2B. Further polymerization within the porogen creates new small polymer globuli that fuse together. However, the conversion is low and only a small number of new globules are formed as most of the monomer is already consumed [25]. With an increase in THF content at the expense of 1-decanol, both the size of the globules and macropores decreases. However, once the macropores become too small, the microglobules fuse together, affecting the homogeneity by the formation of domains with larger agglomerates composed of very small globules (Fig. 2A). Conversely, when the 1-decanol content in the porogen is increased, the local concentration of monomers in the swollen nuclei is higher, and the polymerization reaction mainly proceeds in the swollen nuclei rather than in solution. This facilitates the formation of polymer microglobules that are larger in size. The 
amount of 1-decanol also affects the onset of the phase separation, which is shifted to an earlier conversion. At this stage, the porogen still contains significant amounts of monomer and crosslinker. When the new copolymer is formed, significant in-filling of small pores occurs which causes polymer microglobules to coalesce together. As a result, the individual globules lose their identity and large fused aggregates of microgel particles are formed (see Fig. 2C).

To obtain chromatographic beds with the desired rigidity and mechanical strength for application in ultra-high-pressure flow-through applications, the monoliths were also prepared with a high content of crosslinker. By increasing the DVB content in the polymerization mixture on the account of reducing percentage of $\mathrm{S}$ changes, the crosslinking density of the monolithic backbone increased, and the chemical composition of the monolith changed. Due to the higher reactivity of DVB compared to styrene, more crosslinkers are incorporated in the polymer backbone already at the beginning of the copolymerization reaction. As a result, the nuclei that are formed and phase separated in the initial stage of the polymerization reaction are more densely crosslinked than those formed in a later stage of the process. Structures are now obtained that are similar to those observed while increasing the fraction good solvent in the porogen. An increased percentage of crosslinking monomer leads to an earlier phase separation and formation of highly crosslinked microglobules [28], with reduced tendency to swell with both good porogenic solvent and monomers. As a result, both the globule and macropore sizes decrease as seen in Fig. 1G-I.

To increase the porosity of polymer monoliths different approaches have been described in literature, including the termination of the polymerization process in the early stage of the polymerization reaction and hence steepest part of the conversion curve [29]. To increase the total porosity, we developed monoliths with a monomer to porogen ratio of 25:75 wt $\%$, while 
proceeding the polymerization reaction for $24 \mathrm{~h}$. In addition, initiator content, porogen ratio, and the amount of crosslinker were again systematically varied to obtain monolithic polymers with different pore structure.

\subsection{Assessment of the performance in gradient elution mode}

Initial chromatographic screening of the separation performance of monoliths with different morphologies was carried out using column-scouting runs, using a gradient elution of tryptic digest of cytochrome $c$ at a fixed flow rate $(F=4 \mu \mathrm{L} / \mathrm{min})$, gradient time $\left(t_{G}=10 \min \right)$, and gradient range $(1-40 \%$ B). Fig. 3 shows chromatograms obtained with monolithic columns prepared using a monomer to porogen ratio of 30:70 wt\% and different initiator contents (Fig. 3A-C), porogen ratios (Fig 3D-G), and amounts of crosslinker (Fig. 3H-K). An increase in amount of the initiator from 1 to $3 \mathrm{wt} \%$, with respect to the total monomer content, improved the resolution. Similar as particle size affects the efficiency in packed columns, the efficiency of monolithic stationary phases depends on the size of polymer microglobules and macropores. Hence, reducing these sizes decreased the plate height, but at the expense of column permeability. Furthermore, retention was enhanced due to a larger surface area accessible for the interaction with peptides, resulting from the larger number of smaller globules. Whereas the hydrophilic peptides typically elute at the column dead time when using commercial monolithic columns [12], these peptides were partially resolved using a monolith with improved pore structure and enhanced surface area (Fig. 3B). An increase in the initiator content to $5 \mathrm{wt} \%$ (Fig. 3C) led to a further enhancement in the retention (from $7.58 \mathrm{~min}$ to $8.08 \mathrm{~min}$ for the last eluting peptide). Although the number of smaller globules formed also increased, the column efficiency was significantly impaired. This is most likely related to the inhomogeneity of the monolith, 
affecting the eddy-diffusion contribution to band broadening ( $A$-term). Hence, the initiator content was fixed at $3 \mathrm{wt} \%$ for the remainder of this study.

With increasing the THF-content, monoliths with smaller domain size were formed as observed in the SEM images in Fig. 1D-G. This directly improved the column efficiency as demonstrated in Fig. 3D-F. However, exceeding a certain percentage of THF (e.g., 28.5 wt\%, Fig. 3G) resulted in deteriorated peak widths. This change could be related to the occurrence of domains with larger agglomerates, negatively affecting the structural homogeneity, and thus the $A$-term. Increasing the amount of crosslinker also increased the surface area and improved retention (Fig. 3H-K). This also resulted in a progressive decrease in peak widths, though with a drastic decrease in column permeability.

It should be noted that characterizing monolithic columns with different porous structure while applying a fixed volumetric flow rate (Fig. 3), the mobile-phase velocity $\left(u_{0}\right)$ may vary and the peak width could be affected by the mobile-phase mass transfer contribution $\left(C_{m}\right)$. Bandbroadening parameters $(A, B, C$ term) are typically determined based on isocratic measurement. However, biomolecules, such as peptides and proteins, display a strong dependency of retention in function of the content of organic modifier. As such, a more generic approach is needed to characterize the column performance, which in gradient mode is generally expressed in terms of peak capacity $\left(n_{c}\right)[30]$ :

$n_{c} \approx \frac{t_{G}}{W_{b}}=\frac{t_{G}}{t_{0}} \cdot \frac{\sqrt{L}}{4\left(1+k_{e}\right) \sqrt{H_{\text {grad }}}}$

where $t_{G}$ is the gradient time, $t_{0}$ the column dead time, $W_{b}$ the $4 \sigma$ peak width, $L$ the column length, $k_{e}$ the retention factor at the time of elution, and $H_{\text {grad }}$ the gradient plate height. To assess the effect of flow rate of the gradient plate height, $\sigma$ was determined for synthetic peptides at 
different flow rates while scaling the gradient time proportional to flow rate, i.e., maintaining the gradient steepness $\left(t_{G} / t_{0}\right)$ at 10. Fig. 4A shows chromatograms obtained at three different flow rates as a function of effective retention factor $\left(k_{\text {eff }}=t_{R} / t_{0}-1\right)$. Considering that an identical column length $L=50 \mathrm{~mm}$ was used in all these experiments, the $k_{e}$ for peptide 3 was estimated at different flow rates as reported in [31] using a non-linear retention behaviour relationship (NeueKuss). The gradient plate height could be then determined using the following equation [30]:

$$
H_{\text {grad }}=\frac{\left(\sigma_{t} \cdot u_{0}\right)^{2}}{L\left(1+k_{e}\right)^{2}}
$$

Fig. 4B shows, in analogy to isocratic van Deemter curves, the effect of $u_{0}$ on $H_{\text {grad }}$ for monolithic columns prepared with different porogen composition. Increasing the THF content from 16.5 to $17.5 \mathrm{wt} \%$ leads to a significant improvement in performance in the $C$-term dominated region. In analogy to packed columns, where the $C$-term contribution is proportional to the square of the particle size, a similar dependency could be expected for monolithic columns, considering that the domain size is given as the sum of the microglobule and macropore size. These sizes were estimated from SEM images to be respectively 165 and $530 \mathrm{~nm}$ for the monolith prepared with $16.5 \mathrm{wt} \% \mathrm{THF}$, and 110 and $370 \mathrm{~nm}$ for the monolith prepared using 17.5 wt $\%$ THF in the polymerization mixture. The difference in the slope shown in Fig. 4 cannot be explained only by the effect of domain size on mass transfer. When further reducing the characteristic size by increasing the THF content to $18.5 \mathrm{wt} \%$, the slope of the $C$-term dominated regime increases. In addition, the higher plate-height relation illustrated in Fig. 4 for this monolithic column could readily be explained by a combined significant contribution of eddydiffusion (A-term) induced by inhomogeneity of the monolithic structure. 
To assess the intrinsic quality of the different monolithic columns prepared with different porogen ratios, gradient kinetic plots were constructed taking into account both the peak capacity and the column permeability, see Fig. 5A. The performance (peak capacity, measured based on the average peak width of 5 synthetic peptides and calculated using Eq. 1) at different flow rates was determined at constant gradient slope $\left(t_{G} / t_{0}=10\right)$. Next, the kinetic performance limits, i.e., highest peak capacity in the shortest possible time, were determined using gradient UHPLC conditions and applying Eq. 3 and 4 [32]:

$$
\begin{aligned}
& n_{c, K P L}=1+\left(n_{c}-1\right) \cdot \sqrt{\frac{\Delta P_{\max }-\Delta P_{e x t}}{\Delta P_{c o l}-\Delta P_{e x t}}} \\
& t_{G, K P L}=t_{G, e f f} \cdot \frac{\Delta P_{\max }-\Delta P_{e x t}}{\Delta P_{c o l}-\Delta P_{e x t}}
\end{aligned}
$$

where $\Delta P_{\max }$ is the maximum system pressure ( $\left.80 \mathrm{MPa}\right), \Delta P_{\text {ext }}$ the extra column pressure contribution, and $t_{G, \text { eff }}$ is the effective gradient time that represents the difference in elution time between the first and last eluting peptide $\left(t_{R, \text { last }}-t_{R, f i r s t}\right)$. Hence, Fig. $5 \mathrm{~A}$ represents the sample peak capacity that can be achieved when operating at the kinetic performance limits, and Fig. 5B the corresponding column lengths. The gradient performance limits of the monolithic columns prepared with $16.5 \mathrm{wt} \%$ THF and $18.5 \mathrm{wt} \%$ THF expressed in terms of peak capacity and time do not differ significantly. Although, the latter column contains significantly smaller macropores and microglobules, which results in a decrease in $H_{\text {grad }}$, the column permeability decreases by a factor of 3 affecting the scaling factor $\Delta P_{\max } / \Delta P_{c o l}$. However, to reach the same performance, for example the same peak capacity, such as peak capacity of 100 , the required column length of the monolith with smaller macropores and microglobules (18.5 wt\% THF) can be significantly shorter. The monolithic column prepared with $17.5 \mathrm{wt} \%$ THF produced the best kinetic 
performance. Furthermore, the required column length needed to reach a specific peak capacity is significantly shorter.

\subsection{Potential of monolithic nanomaterials for high-speed gradient separations}

The potential of monolithic nanomaterials was investigated for short gradient at UHPLC conditions, i.e. ultra-fast separations with a run time of around $1 \mathrm{~min}$, providing a high resolution power. To do so, samples of varying complexity were separated on a $50 \mathrm{~mm}$ long column prepared with $17.5 \mathrm{wt} \%$ THF (15 wt $\%$ of both S and DVB, and $3 \mathrm{wt} \%$ AIBN), as presented in Fig. 6. Operated at the maximal system pressure ( $80 \mathrm{MPa}$, coinciding with a flow rate $F=10$ $\mu \mathrm{L} / \mathrm{min}$ ), a $15 \mathrm{~s}$ baseline separation (resolution $R_{s}=1.7$ between peptide 2 and 3 ) could be achieved for the 5 standard peptide mixture (Fig. $5 \mathrm{~A}, n_{c}=18$ ), yielding a peak-production rate $\xi$ $=1.2 \mathrm{~s}^{-1}$. Samples of increasing complexity, such as a tryptic digest of cytochrome $c$ and bovine serum albumin, are shown in Fig.6B-C, respectively. It should be noted that to account for the complexity, gradient run times were increased accordingly, leading to a performance $n_{c}=65$ (for $\left.t_{G}=60 \mathrm{~s}\right)$ and $110\left(t_{G}=3.5 \mathrm{~min}\right)$. These performances were determined based on the separation of the 5 peptide standard using identical chromatographic conditions (i.e. gradient times). This constitutes an increase in peak capacity of $65 \%$ compared to results reported in [12]. Van de Meent reported a peak capacity of 48 using a $50 \mathrm{~mm}$ long monolithic column at a gradient time

of $16.9 \min \left(\xi=2.4 \mathrm{~min}^{-1}\right)$ [33]. To reach a peak capacity of 110 using a column packed with 2 $\mu \mathrm{m}$ silica $\mathrm{C} 18$ particles (also operated at $80 \mathrm{MPa}$ ), a gradient time of approximately $10 \mathrm{~min}$ is required [12]. As can be observed in Fig. 6B showing the separation of the cytochrome $c$ digest, the improved retention properties of the designed column morphology allowed the separation of two additional hydrophilic peaks at the beginning of the gradient (at 23 and $26 \mathrm{~s}$, corresponding 
with the peptides KYIPGTK and YIPGTK), which are generally eluting with the void time if conventional columns would be used [12]. However, even though retention power was increased, additional (even more) hydrophilic peptides are still not retained sufficiently. No significant change in column pressure was observed during prolonged use (> 600 runs), indicating excellent column stability.

To further decrease the globule size and increase the surface area, high porosity monolith were prepared using a monomer-to-porogen ratio of 25:75 wt $\%$. After optimizing the initiator content (5 wt\%), porogen composition (THF/1-decanol), and crosslinking density the monolith shown in SEM micrograph of Fig. 7B was produced. Compared to monolith shown in Fig. 7A (30:70 wt\% monomer-to-porogen), monolith of Fig. 7B consists of even finer globular nanostructure. In order to accommodate for the decrease in column permeability from this later (factor $5, K_{v, 0}=1.6 \times 10^{-15} \mathrm{~m}^{2}$ in contrast to $K_{v, 0}=8.7 \times 10^{-15} \mathrm{~m}^{2}$ for the $30: 70 \mathrm{wt} \%$ monolith from Fig. 7A), and to achieve capillary ballistic gradients [34], short columns with a total length of $25 \mathrm{~mm}$ were prepared and used to separate cytochrome $c$ digest at an equivalent flow rate of 6.0 $\mu \mathrm{L} / \mathrm{min}$, enabled by the operation at $80 \mathrm{MPa}$ of the $25: 75 \mathrm{wt} \%$ monolith, and with a gradient run time of $2.5 \mathrm{~min}$. Using this novel monolithic column, the resolving power for highly hydrophilic peptides eluting at the beginning of the gradient (Fig. 7B) is improved as a result of the larger surface area that contributes to the higher retentive capacity.

\section{Conclusions}

The control that can be exerted over the preparation process of monolithic poly(styreneco-divinylbenzene) monoliths has been applied to facilitate the optimization of nanoporous structures. This control allowed the fine-tuning of both separation efficiency and column 
permeability. Careful optimization of the composition of the polymerization mixture, including porogen composition, percentage of initiator and crosslinker, and monomer to porogen ratio, enabled the preparation of monolithic columns suitable for the efficient separation of biomolecules, including protein digests. In analogy to packed columns, decreasing the characteristic size, i.e., the domain size given as the combined size of the macropore and microglobule, led to a reduction in the gradient plate height. However, gradient van Deemter curves plotted for monolithic columns prepared with different porogen compositions have demonstrated that the eddy-diffusion induced by structural inhomogeneity is the key contributor to the plate-height size. Hence, more insights in the phase separation mechanism in the presence of complex binary porogen multi-monomer polymerization mixtures are required. Alternatively, a viable route to improve the homogeneity of monolithic nanostructures could be the combination of structure directing agents, such as micropillars in chips, together with carefully optimized polymerization mixtures [35].

\section{Acknowledgements}

Support of this work by a grant of the Research Foundation Flanders (G.0919.09) is gratefully acknowledged. Part of this work was performed by F.S. at the Molecular Foundry, Lawrence Berkeley National Laboratory supported by the Office of Science, Office of Basic Energy Sciences, Scientific User Facilities Division of the U.S. Department of Energy, under Contract No. DE-AC02-05CH11231.

\section{References}


[1] A. Tisselius, Chromatographic analysis - General introduction, Disc. Faraday Soc. 7 (1949) 7-11.

[2] S. Hjérten, J.L. Liao, R. Zhang, High-performance liquid-chromatography on continuous polymer beds, J. Chromatogr. 473 (1989) 1298-1302.

[3] T.N. Tennikova, B.G. Belenkii, F. Svec, High-performance membrane chromatography; a novel method of protein separation, J. Liquid Chromatogr. 13 (1990) 63-70.

[4] J. Lukas, F. Svec, J. Kalal, Polar polymeric sorbents based on glycidyl methacrylate copolymers, J. Chromatogr. 153 (1978) 15-22.

[5] I. Gusev, X. Huang, C. Horváth, Capillary columns with in situ formed porous monolithic packing for micro high-performance liquid chromatography and capillary electrochromatography, J. Chromatogr. A 855 (1999) 273-290.

[6] M. Petro, F. Svec, J.M.J. Fréchet, Molded continuous poly(styrene-co-divinylbenzene) rod as a separation medium for the very fast separation of polymers, J. Chromatogr. A 752 (1996) 59-66.

[7] S. Xie, R.W. Allington, F. Svec, J.M.J. Fréchet, Rapid reversed-phase separation of proteins and peptides using optimized 'moulded' monolithic poly(styrene-codivinylbenzene) columns, J. Chromatogr. A 865 (1999) 169-174.

[8] C.G. Huber, A. Krajete, Analysis of nucleic acids by capillary ion-pair reversed-phase HPLC coupled to negative-ion electrospray ionization mass spectrometry, Anal. Chem. 71 (1999) 3730-3739.

[9] C.G. Huber, G. Kleindienst, G.K. Bonn, Application of micropellicular polystyrene/divinylbenzene stationary phases for high-performance reversed-phase liquid chromatography electrospray-mass spectrometry of proteins and peptides, Chromatographia 44 (1997) 438-448. 
[10] S. Eeltink, S. Dolman, F. Detobel, R. Swart, M. Ursem, P.J. Schoenmakers, Highefficiency liquid chromatography-mass spectrometry separations with $50 \mathrm{~mm}, 250 \mathrm{~mm}$, and $1 \mathrm{~m}$ long polymer-based monolithic capillary columns for the characterization of complex proteolytic digests, J. Chromatogr. A 1217 (2010) 6610-6615.

[11] S. Eeltink, B. Wouters, G. Desmet, M. Ursem, D. Blinco, G.D. Kemp, A. Treumann, High-resolution separations of protein isoforms with liquid chromatography time-of-flight mass spectrometry using polymer monolithic capillary columns, J. Chromatogr. A 1218 (2011) 5504-5511.

[12] A. Vaast, L. Nováková, G. Desmet, B. de Haan, R. Swart, S. Eeltink, High-speed gradient separations of peptides and proteins using polymer-monolithic poly(styrene-codivinylbenzene) capillary columns at ultra-high pressure, J. Chromatogr. A 1304 (2013) $177-182$.

[13] C. Viklund, F. Svec, J.M.J. Fréchet, Monolithic, "molded", porous materials with high flow characteristics for separations, catalysis, or solid-phase chemistry: control of porous properties during polymerization, Chem. Mater. 8 (1996) 744-750.

[14] E. Byström, C. Viklund, K. Irgum, Differences in porous characteristics of styrenic monoliths prepared by controlled thermal polymerization in molds of varying dimensions, J. Sep. Sci. 33 (2010) 191-199.

[15] Q.C. Wang, F. Svec, J.M.J. Fréchet, Macroporous polymeric stationary-phase rod as continuous separation medium for reversed-phase chromatography, Anal. Chem. 65 (1993) 2243-2248.

[16] B.P. Santora, M.R. Gagné, K.G. Moloy, N.S. Radu, Porogen and cross-linking effects on the surface area, pore volume, distribution, and morphology of macroporous polymers obtained by bulk polymerization, Macromolecules 34 (2001) 658 . 
[17] O. Okay, Macroporous copolymer networks, Prog. Polym. Sci. 25 (2000) 711-779.

[18] Y. Huo, P.J. Schoenmakers, W. Th. Kok, Efficiency of methacrylate monolithic columns in reversed-phase liquid chromatographic separations, J. Chromatogr. A 1175 (2007) 8188.

[19] C. Stassen, G. Desmet, K. Broeckhoven, L. Van Lokeren, S. Eeltink, Characterization of polymer monolithic columns for small-molecule separations using total pore blocking, J. Chromatogr. A 1325 (2014) 115-120.

[20] H. Poppe, Some reflections on speed and efficiency of modern chromatographic methods, J. Chromatogr. A 778 (1997) 3-21.

[21] J.H. Knox, High Speed Liquid Chromatography, Ann. Rev. Phys. Chem. 24 (1973) 2949.

[22] G. Desmet, D. Clicq, P. Gzil, Geometry-independent plate height representation methods for the direct comparison of the kinetic performance of LC supports with a different size or morphology, Anal. Chem. 77 (2005) 4058-4070.

[23] T.J. Causon, R.A. Shellie, E.F. Hilder, Kinetic performance appraisal of poly(styrene-codivinylbenzene) monolithic high-performance liquid chromatography column for biomolecule analysis, J. Chromatogr. A 1217 (2010) 3765-3769.

[24] G. Desmet, D. Cabooter, P. Gzil, H. Verelst, D. Mangelings, Y. Vander Heyden, D. Clicq, Future of high pressure liquid chromatography: do we need porosity or do we need pressure?, J. Chromatogr. A 1130 (2006) 158-166.

[25] S. Wouters, B. Wouters, A. Vaast, H. Terryn, G. Van Assche, S. Eeltink, Monitoring the morphology development of polymer-monolithic stationary phases by thermal analysis, J. Sep. Sci. 37 (2014) 179-186. 
[26] D.C. Sherrington, Preparation, structure and morphology of polymer supports, Chem. Commun. 21 (1998) 2275-2286.

[27] S.H. Lubbad, M.R. Buchmeiser, Highly cross-linked polymeric capillary monoliths for the separation of low, medium, and high molecular weight analytes, J. Sep. Sci. 32 (2009) 2521-2529.

[28] A.S. Zalusky, R. Olayo-Valles, C.J. Taylor, M.A. Hillmyer, Mesoporous polystyrene monoliths, J. Am. Chem. Soc. 123 (2001) 1519-1520.

[29] A. Greiderer, L. Trojer, C.W. Huck, G.K. Bonn, Influence of the polymerization time on the porous and chromatographic properties of monolithic poly(1,2-bis(pvinylphenyl)ethane capillary columns, J. Chromatogr. A 1216 (2009) 7747-7754.

[30] U.D. Neue, D.H. Marchand, L.R. Snyder, Peak compression in reversed-phase gradient elution, J. Chromatogr. A 1111 (2006) 32-39.

[31] A. Vaast, E. Tyteca, G. Desmet, P.J. Schoenmakers, S. Eeltink, Gradient-elution parameters in capillary liquid chromatography for high-speed separations of peptides and intact proteins, J. Chromatogr. A 1355 (2014) 149-157.

[32] A. Vaast, K. Broeckhoven, S. Dolman, G. Desmet, S. Eeltink, Comparison of the gradient kinetic performance of silica monolithic capillary columns with columns packed with 3 $\mu \mathrm{m}$ porous and $2.7 \mu \mathrm{m}$ fused-core silica particles, J. Chromatogr. A 1228 (2012) 270-275.

[33] M.H. van de Meent, S. Eeltink, G.J. de Jong, Potential of poly(styrene-co-divinylbenzene) monolitic columns for the LC-MS analysis of protein digests, Anal. Bioanal. Chem. 399 (2011) 1845-1852. 
[34] L.A. Romanyshyn, P.R. Tiller, Ultra-short columns and ballistic gradients: considerations for ultra-fast chromatographic liquid chromatographic - tandem mass spectrometric analysis, J. Chromatogr. A 928 (2001) 41-51.

[35] F. Detobel, H. Eghbali, S. De Bruyne, H. Terryn, H. Garderniers, G. Desmet, Effect of the presence of an ordered micro-pillar array on the formation of silica monoliths, J. Chromatogr. A 1216 (2009) 7360-7367.

\section{Figure captions}

Figure 1. Scanning electron micrographs of monolithic poly(styrene-co-divinylbenzene) columns prepared by varying the amount of $(i)$ initiator: (A) 1 , (B) 5, and (C) 9 wt $\%$ AIBN with respect to the monomer content. Composition of the polymerization mixture was $15 \mathrm{wt} \% \mathrm{~S}, 15 \mathrm{wt} \% \mathrm{DVB}$, $17.5 \mathrm{wt} \%$ THF, and $52.5 \mathrm{wt} \%$ 1-dec; (ii) the porogen composition: (D) 14, (E) 16, and (F) 18 
wt $\%$ THF, in the polymerization mixture consisting of $3 \mathrm{wt} \%$ AIBN with respect to the monomer content of, $15 \mathrm{wt} \% \mathrm{~S}, 15 \mathrm{wt} \% \mathrm{DVB}$, and a $70 \%$ total porogen content; (iii) the crosslinking density: (G) 20, (H) 25, and (I) $30 \mathrm{wt} \%$ DVB used in a polymerization mixture containing a total of $30 \%$ monomers (S+DVB), $3 \mathrm{wt} \%$ AIBN with respect to the monomers, 17.5 $\mathrm{wt} \% \mathrm{THF}$ and $52.5 \mathrm{wt} \%$ 1-dec.

Figure 2. Schematic of the effect of porogen composition on connectivity and polymer microglobule size in a macroporous monolithic network. When the amount of 'good' solvent (THF) in the porogen is decreased (from A to C) at the expense of 'bad' solvent (1-decanol), an increase in polymer microglobule and macropore size is observed. At high concentrations of THF, small microglobules will tend to coalesce together to form inhomogeneously dispersed agglomerates.

Figure 3. Gradient separation of a cytochrome $c$ digest using a fixed flow rate of $4 \mu \mathrm{L} / \mathrm{min}$, a gradient range of $1-40 \% \mathrm{~B}$, and a gradient time of 10 min using monolithic columns with different morphologies prepared by varying: $(i)$ the initiator concentration: $(\mathbf{A}) 1,(\mathbf{B}) 3$, and $(\mathbf{C}) 5$ wt $\%$ AIBN used in a polymerization mixture containing 15 wt $\%$ S, 15 wt $\%$ DVB, 17.5 wt $\%$ THF, and $52.5 \mathrm{wt} \%$ 1-dec; (ii) the porogen composition (D) 15.5, (E) 16.5, (F) 17.5, and (G) $18.5 \mathrm{wt} \% \mathrm{THF}$, used in a polymerization mixture containing $3 \mathrm{wt} \%$ AIBN with respect to the monomer content consisting of $15 \mathrm{wt} \% \mathrm{~S}, 15 \mathrm{wt} \%$ DVB and a $70 \%$ total porogen content; and (iii) the crosslinking density: (H) 15, (I) 20, (J) 25, (K) $30 \mathrm{wt} \%$ DVB, used in a polymerization 
mixture containing a total of $30 \%$ monomers $(\mathrm{S}+\mathrm{DVB}), 3 \mathrm{wt} \%$ AIBN with respect to the monomers, $17.5 \mathrm{wt} \%$ THF and $52.5 \mathrm{wt} \%$ 1-dec.

Figure 4. Gradient performance of the monolithic columns based on the separation of a mixture of 5 synthetic peptides carried out at different flow rates while maintaining a constant gradient slope $\left(t_{G} / t_{0}=10\right)$. (A) Chromatograms as a function of effective retention factor $\left(k_{e f f}=t_{R} / t_{0}-1\right)$, (B) Effect of mobile-phase velocity on gradient plate height for monolithic columns prepared with different porogen compositions $16.5 \mathrm{wt} \%$ THF (closed squares $\mathbf{0}$ ), $17.5 \mathrm{wt} \%$ THF (closed diamonds $\diamond$ ), and $18.5 \mathrm{wt} \%$ THF (open red circles $\mathrm{O}$ )

Figure 5. Effect of porogen composition on the gradient kinetic performance limits: sample peak capacity vs. time (A), and corresponding column lengths (B). Monolithic columns prepared with 16.5 wt $\%$ THF (closed squares $\square$ ), 17.5 wt $\%$ THF (closed diamonds $\bullet$ ), and 18.5 wt\% THF (open red circles $\mathrm{O}$ ). All the plots were extrapolated to a maximal pressure of $80 \mathrm{MPa}$.

Figure 6. High-resolution peptide separations, carried out with the in-house prepared $50 \mathrm{~mm} \times$ $200 \mu \mathrm{m}$ i.d. organic polymer monolithic column, using polymerization mixture consisting of 3 wt $\%$ AIBN with respect to the total monomer content of $30 \%$ (15 wt $\%$ of both S and DVB), $70 \%$ porogen. The separations were performed at $60^{\circ} \mathrm{C}$, using a flow rate $F=10 \mu \mathrm{L} / \mathrm{min}$, corresponding to a total pressure drop of 79.5 MPa. To account for the sample complexity of (A) 5 peptide standard $\left(t_{G}=15 \mathrm{~s}, n_{c}=18\right),(\mathbf{B})$ tryptic digest of cytochrome $c\left(t_{G}=60 \mathrm{~s}, n_{c}=65\right)$, and (C) tryptic digest of bovine serum albumin $\left(t_{G}=210 \mathrm{~s}, n_{c}=110\right)$, gradient times were adjusted 
respectively. The baseline separation of the 5 peptide sample in (A) in $15 \mathrm{~s}$ is characterized with a resolution of 1.7 between peptide 2 and 3 . The peak capacities reported for $(\mathbf{B})$ and $(\mathbf{C})$ are based on identical runs for the separation of a 5 peptide sample.

Figure 7. SEM micrographs of monolithic columns and corresponding gradient separations of cytochrome $c$ digest applying UHPLC conditions $(\Delta P=80 \mathrm{MPa})$ on $200 \mu \mathrm{m} \times 25 \mathrm{~mm}$ columns based on a polymerization mixture consisting of (A-B) $3 \mathrm{wt} \%$ AIBN with respect to the total monomer content of $30 \%$ (15 wt $\%$ of both S and DVB), $70 \%$ porogen, and (C-D) a mixture consisting of $5 \mathrm{wt} \%$ AIBN with respect to a total monomer content of $25 \%$ and $75 \%$ porogen. 

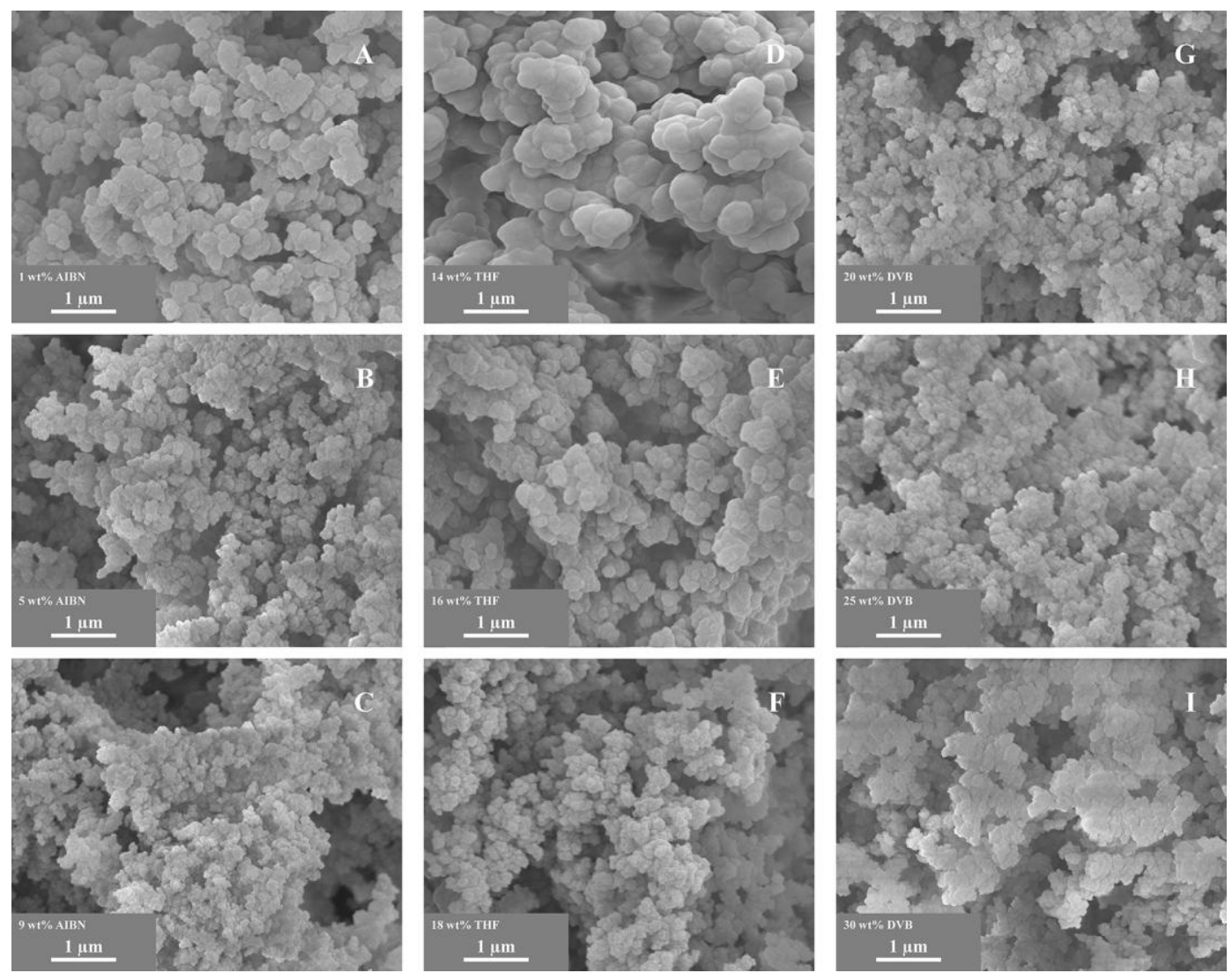


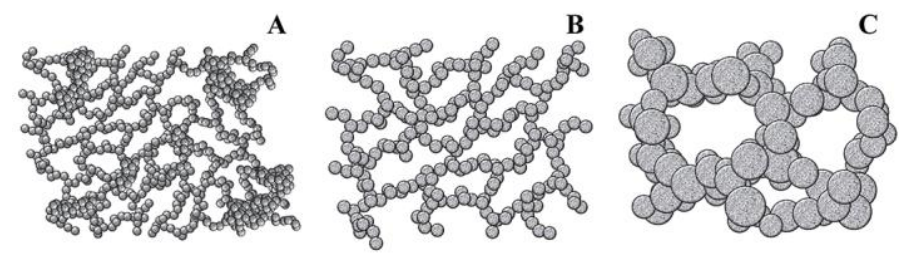



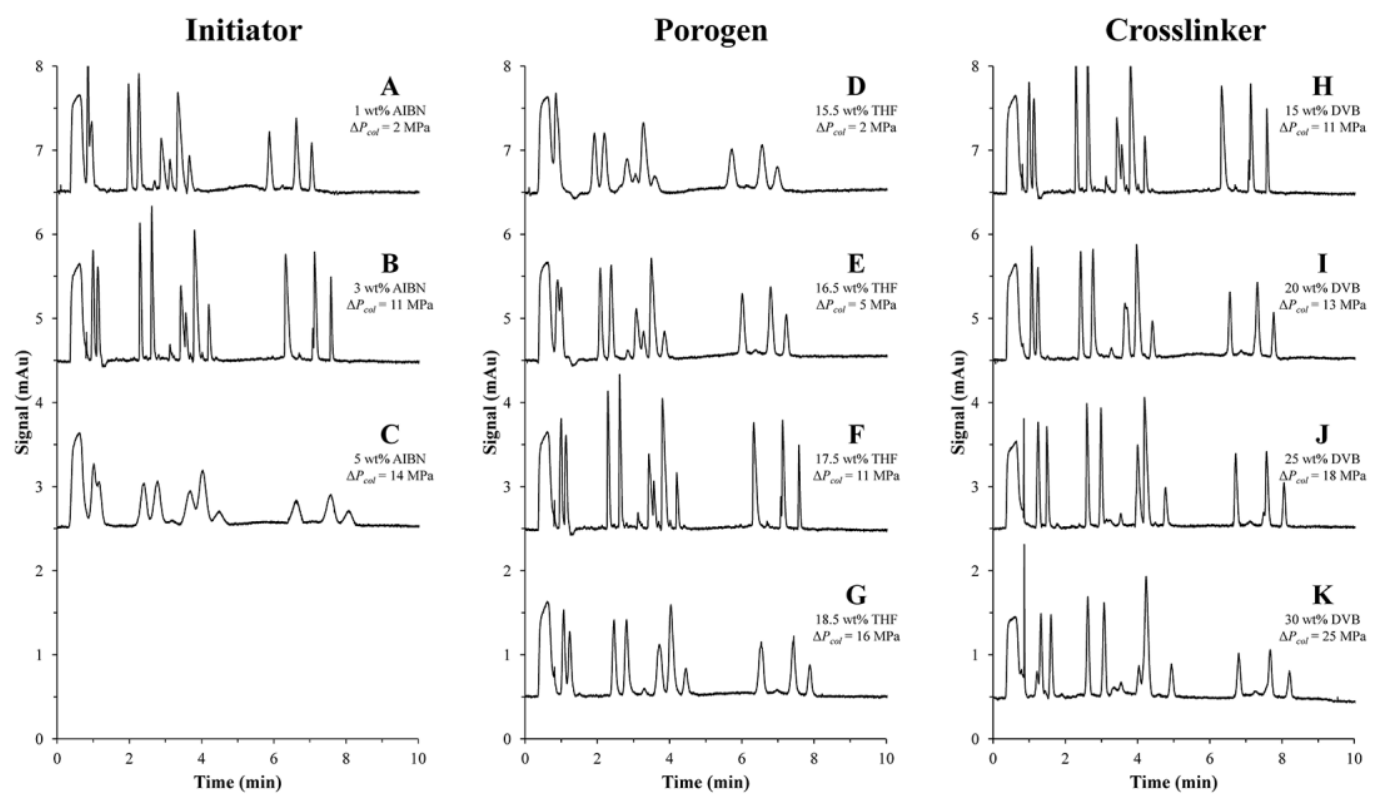

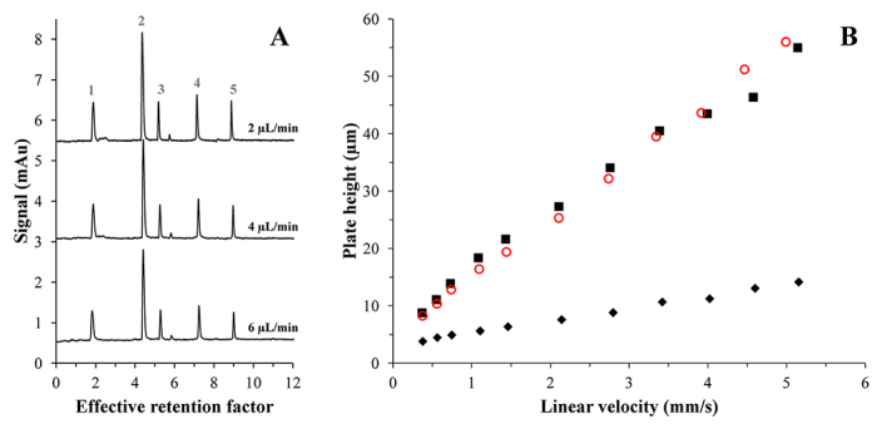

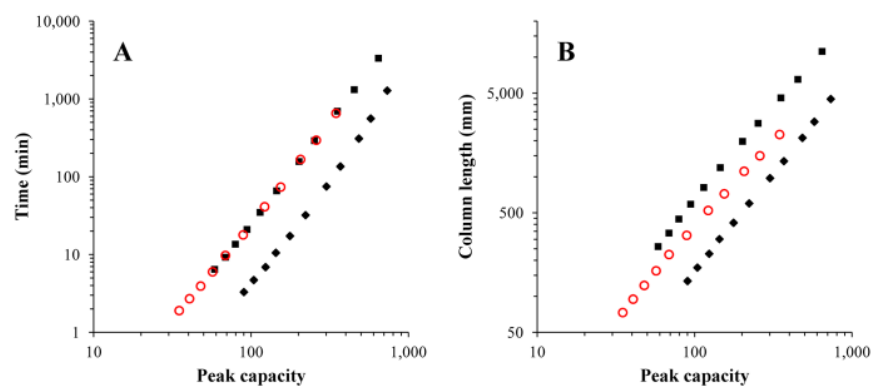

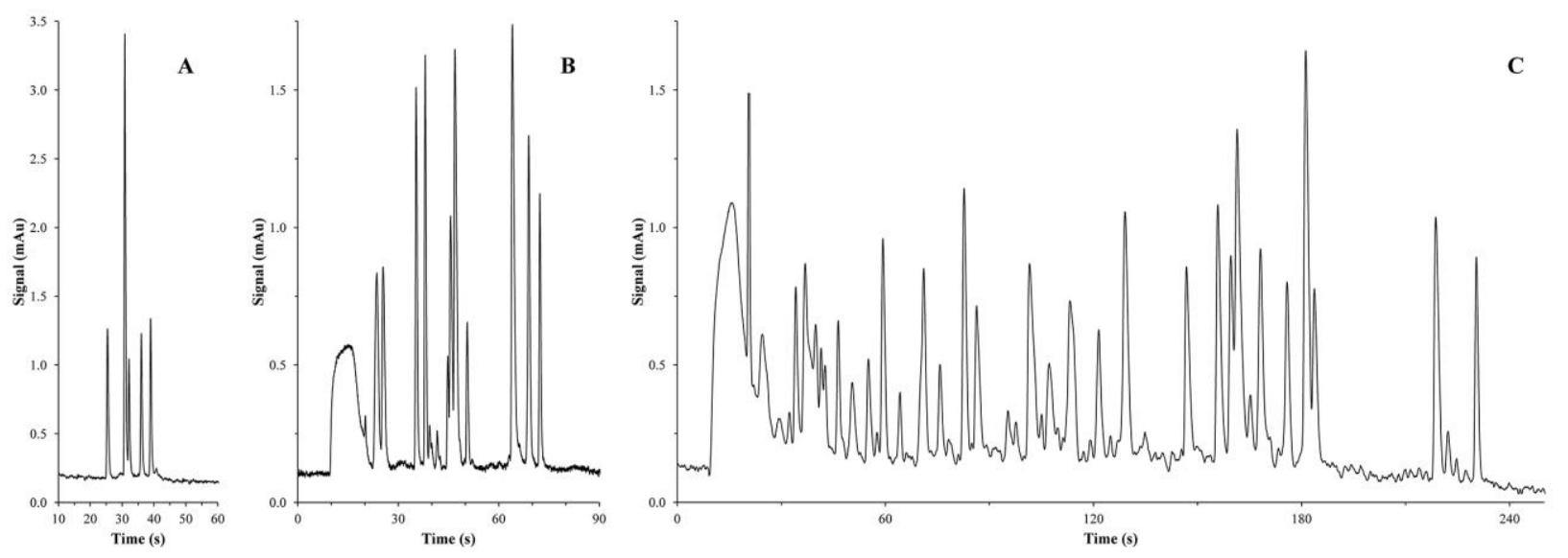

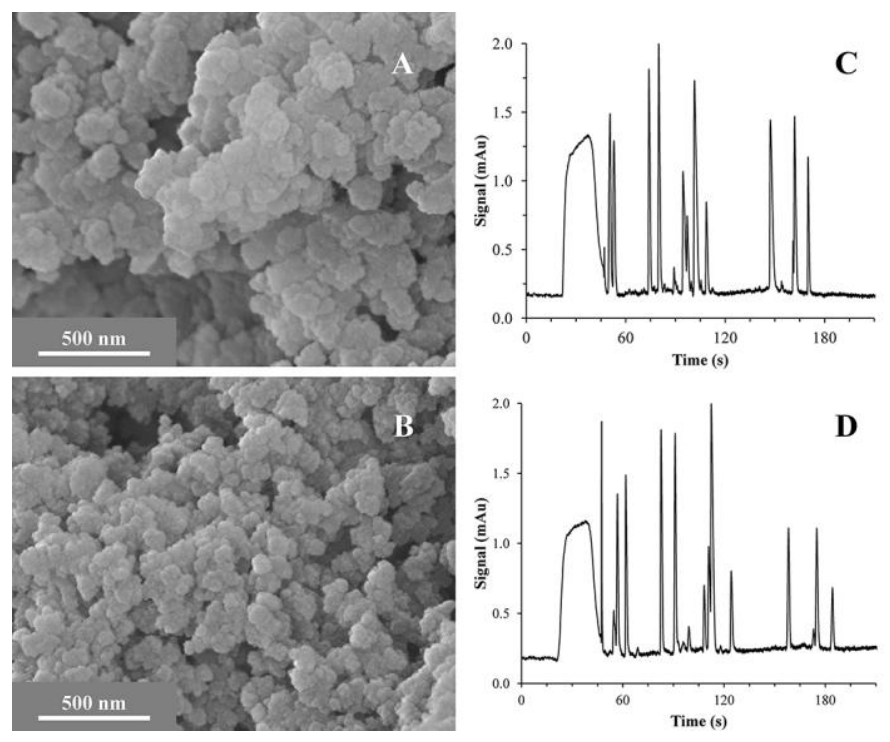